\title{
Reliability and reproducibility of sciatic nerve magnetization transfer imaging and $\mathrm{T} 2$ relaxometry
}

\author{
Fabian Preisner ${ }^{1} \cdot$ Rouven Behnisch ${ }^{2} \cdot$ Olivia Foesleitner $^{1} \cdot$ Daniel Schwarz ${ }^{1} \cdot$ Michaela Wehrstein $^{3} \cdot$ Hagen Meredig $^{1}$. \\ Birgit Friedmann-Bette $^{3} \cdot$ Sabine Heiland $^{1} \cdot$ Martin Bendszus $^{1} \cdot$ Moritz Kronlage $^{1}$ (D)
}

Received: 22 January 2021 / Revised: 8 April 2021 / Accepted: 11 May 2021 / Published online: 9 June 2021

(C) The Author(s) 2021

\begin{abstract}
Objectives To assess the interreader and test-retest reliability of magnetization transfer imaging (MTI) and T2 relaxometry in sciatic nerve MR neurography (MRN).

Materials and methods In this prospective study, 21 healthy volunteers were examined three times on separate days by a standardized MRN protocol at 3 Tesla, consisting of an MTI sequence, a multi-echo T2 relaxometry sequence, and a highresolution T2-weighted sequence. Magnetization transfer ratio (MTR), T2 relaxation time, and proton spin density (PSD) of the sciatic nerve were assessed by two independent observers, and both interreader and test-retest reliability for all readout parameters were reported by intraclass correlation coefficients (ICCs) and standard error of measurement (SEM).

Results For the sciatic nerve, overall mean \pm standard deviation MTR was $26.75 \pm 3.5 \%$, T2 was $64.54 \pm 8.2 \mathrm{~ms}$, and PSD was $340.93 \pm 78.8$. ICCs ranged between 0.81 (MTR) and 0.94 (PSD) for interreader reliability and between 0.75 (MTR) and 0.94 (PSD) for test-retest reliability. SEM for interreader reliability was 1.7\% for MTR, $2.67 \mathrm{~ms}$ for T2, and 21.3 for PSD. SEM for test-retest reliability was $1.7 \%$ for MTR, $2.66 \mathrm{~ms}$ for T2, and 20.1 for PSD.

Conclusions MTI and T2 relaxometry of the sciatic nerve are reliable and reproducible. The values of measurement imprecision reported here may serve as a guide for correct interpretation of quantitative MRN biomarkers in future studies.

Key Points

- Magnetization transfer imaging (MTI) and T2 relaxometry of the sciatic nerve are reliable and reproducible.

- The imprecision that is unavoidably associated with different scans or different readers can be estimated by the here presented SEM values for the biomarkers T2, PSD, and MTR.

- These values may serve as a guide for correct interpretation of quantitative MRN biomarkers in future studies and possible clinical applications.
\end{abstract}

Keywords Magnetic resonance imaging $\cdot$ Peripheral nervous system $\cdot$ Biomarkers $\cdot$ Observer variation $\cdot$ Reproducibility of results

\section{Abbreviations}

DTI Diffusion tensor imaging

ICC Intraclass correlation coefficient

Moritz Kronlage

Moritz.Kronlage@med.uni-heidelberg.de

1 Department of Neuroradiology, Heidelberg University Hospital, Im Neuenheimer Feld 400, 69120 Heidelberg, Germany

2 Institute of Medical Biometry and Informatics, University of Heidelberg, Im Neuenheimer Feld 130.3, 69120 Heidelberg, Germany

3 Department of Sports Medicine (Internal Medicine VII), Medical Clinic, Heidelberg University Hospital, Im Neuenheimer Feld 410, 69120 Heidelberg, Germany
MDD Minimum detectable difference

MRN MR neurography

MTI Magnetization transfer imaging

MTR Magnetization transfer ratio

PNS Peripheral nervous system

PSD Proton spin density

SEM Standard error of measurement

\section{Introduction}

High-resolution magnetic resonance neurography (MRN) has emerged to a useful diagnostic tool for various neuropathies and allows detecting minor damage of the peripheral nervous 
system (PNS) with high sensitivity [1-6]. In a clinical setting, MRN is typically based on visual assessment of nerve lesions using high-resolution T2-weighted sequences.

Morphological nerve imaging can be complemented by quantitative MRI techniques, such as diffusion tensor imaging (DTI), which are increasingly studied in traumatic, hereditary, inflammatory, and degenerative neuropathies [7-9]. These techniques offer additional information about nerve microstructure or tissue composition and provide quantitative biomarkers. In addition to DTI — which has been well evaluated [10] - T2 relaxometry and magnetization transfer imaging (MTI) have increasingly been applied as novel quantitative MRN techniques in recent investigations [11-14].

$\mathrm{T} 2$ relaxometry yields the readout parameters transverse relaxation time (T2) and proton spin density (PSD) and is conducted by a multi-echo sequence and fitting of an exponential function. MR signal loss after a radiofrequency pulse usually follows an exponential decay characterized by the tissuespecific time constant $\mathrm{T} 2$, which describes the time in which the transverse magnetization decreases to $37 \%$ (1/e) of its initial value $[15,16]$. PSD is another tissue-intrinsic parameter and refers to the concentration of protons excitable by MRI [17]. It equals the theoretical MR-signal-intensity without any effects of transverse relaxation. While T2 is considered a biomarker of free water, PSD is regarded to reflect total water content including protons bound to macromolecules such as myelin [16-18].

MTI is an MRI technique that generates the readout parameter magnetization transfer ratio (MTR) [19-21]. It relies on the principle that protons bound to macromolecules may be selectively saturated by an off-resonance radiofrequency pulse since resonance occurs in a larger bandwidth off the Larmor frequency in these bound protons compared to free-water protons. Magnetic saturation is then transferred to free-water protons which leads to a lower MR signal in a sequence with a saturation pulse than without. MTI works by a pair of MR sequences, one with and one without a preceding off-resonance saturation pulse. By calculating the relative difference between signals, the MTR can be determined, which reflects the concentration of bound protons and their interaction with protons in free water and is considered a biomarker of demyelination [22, 23].

Recent studies suggested that $\mathrm{T} 2$ relaxometry and MTI could yield promising MRN biomarkers for the assessment of various neuropathies [24-27]. However, these techniques have not been implemented into clinical routine yet. To use these parameters for decisions in individual patients, it is crucial to assess reliability by a quantitative estimation of the measurement error attributed to different examinations (testretest) or different readers (interreader). First studies implemented reliability analyses of these techniques in their investigations with promising results, but a systematic assessment of both test-retest and interreader reliability in a larger cohort and a quantification of the measurement error are still lacking $[11,28,29]$.
Reliability is commonly expressed by the intraclass correlation coefficient (ICC), a dimensionless parameter ranging between 0 and 1 [30]. However, the ICC should be interpreted cautiously since it depends not only on the measurement error but also on the sample variance. To describe measurement imprecision independently of the sample variance, the standard error of measurement (SEM) may be calculated, which indicates how test results spread around a "true" value [31, 32]. Besides, the SEM allows calculating the minimum detectable difference (MDD), which is the smallest difference needed between separate measurements in order for the difference to be considered real [31,32]. Although sometimes sharing the same abbreviation, the standard error of measurement should not be confused with the standard error of the mean, which is considered a different statistical parameter.

The aim of the present study was to systematically assess interreader and test-retest reliability of MTI and T2 relaxometry in MRN and to quantify the measurement accuracy by means of ICC, SEM, and MDD. Being the most commonly examined and well-accessible nerve, we focused on the sciatic nerve and examined a cohort of 21 healthy participants who underwent three MRN examinations on separate days which were analyzed by two readers independently.

\section{Materials and methods}

This prospective study was approved by the institutional ethics board and written informed consent was obtained from all participants prior to the examinations. The study design is summarized in Fig. 1.

\section{Study subjects}

Twenty-one healthy adult ( $>18$ years) male volunteers were prospectively enrolled over a period of 22 months. Mean age was $24.1 \pm 3$ years (range: $20-30$ years), mean weight was $76.8 \pm 6.7 \mathrm{~kg}$, mean height was $1.81 \pm 0.1 \mathrm{~m}$, and mean body mass index was $23.4 \pm 1.7 \mathrm{~kg} / \mathrm{m}^{2}$. Exclusion criteria were history of peripheral nerve disorders and general contraindications concerning MRI.

\section{MR data acquisition}

All participants were prospectively examined at a 3-Tesla MR scanner (Magnetom Prisma-FIT, Siemens Healthineers) on three separate days using a 15-channel transmit-receive knee coil (Siemens Healthineers). The mean timespan was $5.7 \pm 2.7$ days between scans 1 and 2 and $4.8 \pm 0.8$ days between scans 2 and 3. MRN of the sciatic nerve of the dominant leg was conducted at mid to distal thigh level according to the following protocol (MR sequence parameters in Table 1): 


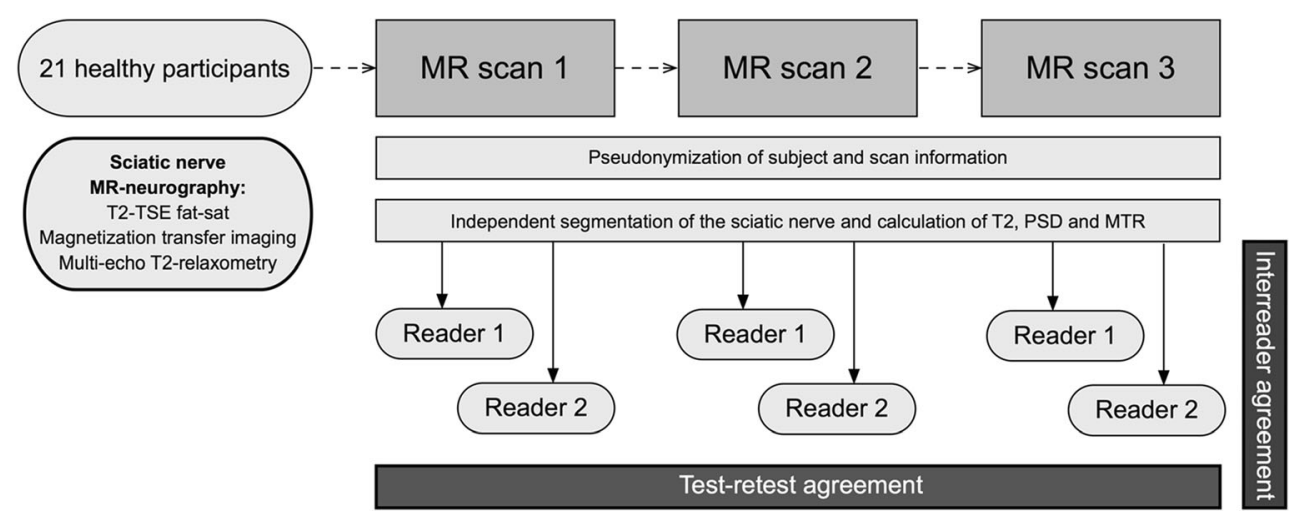

Fig. 1 Study design flowchart. Twenty-one healthy participants underwent repeated MR neurography of their sciatic nerve on three separate days, each covering the exact same anatomical region. Image analysis and postprocessing were performed by two independent readers.

1. An axial $\mathrm{T} 2$-weighted 2-dimensional turbo spin-echo (TSE) sequence to provide anatomical coverage with a high structural resolution,

2. An axial 2-dimensional multi-spin-echo (MSE) sequence for $\mathrm{T} 2$ relaxometry, and

3. Two-axial proton density-weighted 3-dimensional gradient-echo (GRE) FLASH sequences with and without an off-resonance saturation pulse (Gaussian envelop, duration $9984 \mu$ s, frequency offset $1200 \mathrm{~Hz}$ ) at the exact same slice position for MTI. An adaptive inline image filter (Siemens Healthineers) was applied to reduce possible effects of B1 inhomogeneities on the received signal.

\section{Image postprocessing}

Image postprocessing and segmentation were performed within 3 weeks after completion of image acquisition. First, images were visually assessed for motion artifacts and other artifacts that might impede segmentation of the sciatic nerve or that might modify readout parameters by projection on nervous tissue. All images were analyzed by two readers
Interreader agreement and test-retest reproducibility were statistically assessed for each of the biomarkers transverse relaxation time (T2), proton spin density (PSD), and magnetization transfer ratio (MTR)

M.K. and F.P. with more than 6 and 4 years of experience in neuromuscular imaging, respectively, using the DICOMviewer OsiriX (Pixmeo Sarl). Six central slices within each image slab covering the same anatomical region were identified by F.P. prior to further analysis. Manual nerve segmentation was subsequently performed in the high-resolution T2weighted images by both readers independently and was restricted to the tibial portion of the sciatic nerve to prevent inclusion of paraneurial fat (for representative examples of image postprocessing, see Fig. 2) [33].

\section{$\mathrm{T} 2$ relaxometry}

T2 relaxometry was based on MR signal analysis of the sciatic nerve in the MSE sequence. ROIs from the T2-weighted images were copied onto the corresponding MSE slice with TE = $20 \mathrm{~ms}$, in which the boundaries of the sciatic nerve could be delineated best. Manual correction of distortion artifacts was performed by each reader independently, if necessary. Signal intensity for the whole ROI was determined for every echo time using the OsiriX plugin ROI enhancement, since averaging over all voxels helps to denoise data. Then, an exponential function was fitted:
Table 1 MRN sequence parameters

\begin{tabular}{llll}
\hline & T2 TSE & T2 relaxometry & Magnetization transfer imaging \\
\hline TR/TE $(\mathrm{ms})$ & $8640 / 54$ & $2400 / 10,20,30 \ldots 120$ & $61 / 4.92$ \\
FOV $\left(\mathrm{mm}^{2}\right)$ & $160 \times 160$ & $160 \times 160$ & $160 \times 160$ \\
Matrix size & $512 \times 333$ & $192 \times 169$ & $128 \times 128$ \\
Slice thickness $(\mathrm{mm})$ & 3.5 & 3.5 & 4 \\
Interslice gap $(\mathrm{mm})$ & 0.35 & 0.35 & - \\
No. of slices & 41 & 13 & 18 \\
Flip angle $\left({ }^{\circ}\right)$ & 150 & 180 & 7 \\
Fat saturation & Yes $(\mathrm{spectral})$ & Yes $($ spectral) & Yes (spectral) \\
Acquisition time & 6 min $48 \mathrm{~s}$ & 6 min $50 \mathrm{~s}$ & 1 min 56 s each $($ on + off $)$ \\
\hline
\end{tabular}




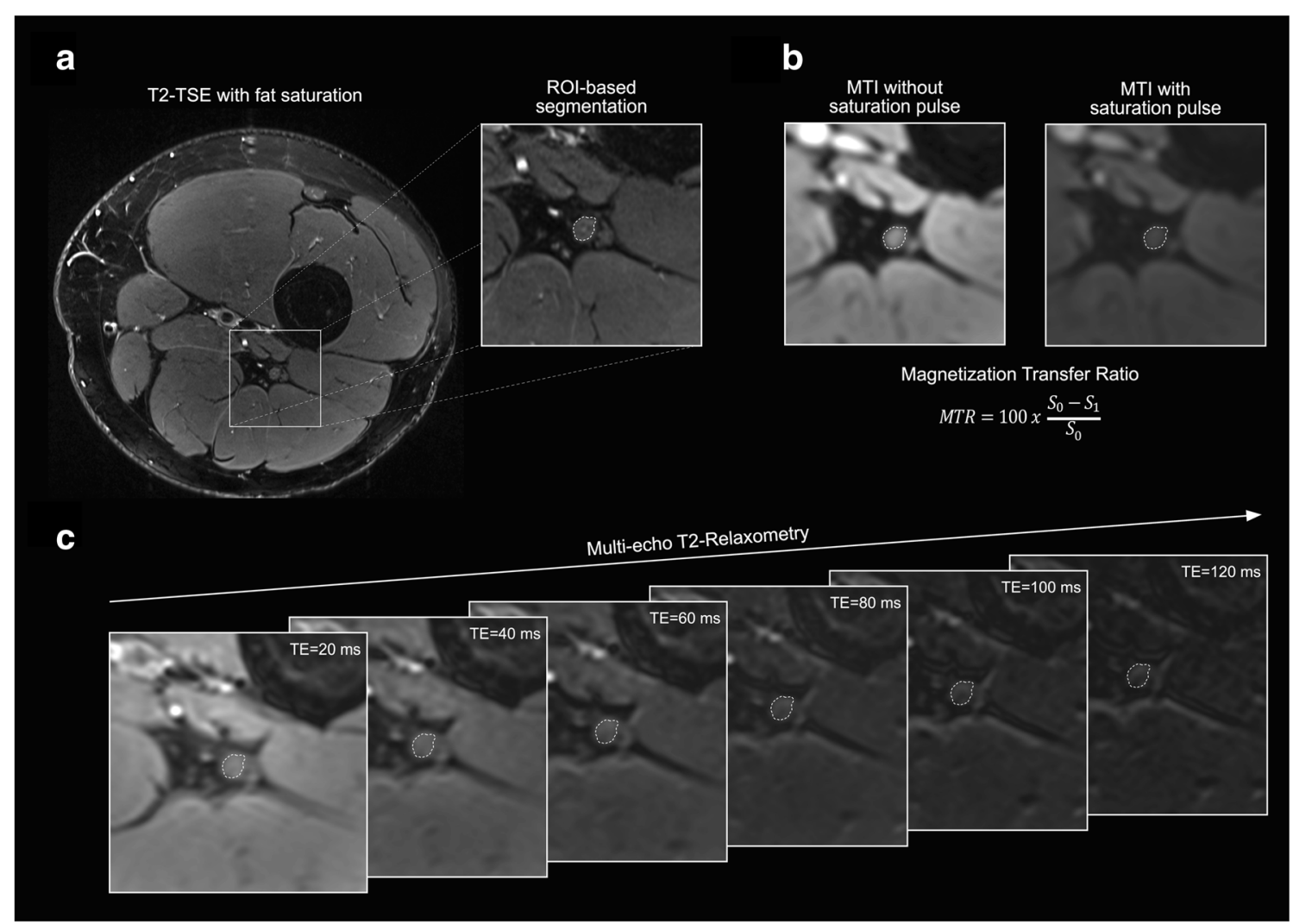

Fig. 2 Image postprocessing. Nerve segmentation was performed by each reader independently by delineating the tibial portion of the sciatic nerve using the freehand region of interest (ROI) tool in Osirix in the T2turbo spin-echo (TSE) sequence (a). Subsequently, ROIs were transferred onto co-registered gradient-echo sequences for magnetization transfer imaging (MTI) (b), and multi-echo sequences for T2-relaxometry (c) and quantitative readout parameters were assessed. MTR $=$ magnetization transfer ratio, $\mathrm{TE}=$ echo time, $\mathrm{S}_{0}=\mathrm{MR}$ signal intensity without saturation pulse, $S_{1}=$ MR signal intensity with saturation pulse
$S(T E)=\mathrm{PSD} \times \mathrm{e}^{-\frac{T E}{T 2}}+$ offset,

as described previously [26, 33, 34], where S(TE) stands for the signal intensity at a given echo time TE, PSD is a dimensionless value for proton spin density, and T2 is the transverse relaxation time. To minimize systematic error, we restricted the analysis to the even echoes and used the offset as a fitting parameter, as described previously [34-36]. Additionally, a normalized PSD was calculated by dividing the $\mathrm{PSD}_{\text {nerve }}$ by a PSD of skeletal muscle ( $\mathrm{PSD}_{\text {nerve }} / \mathrm{PSD}_{\text {muscle }}$ ). The ROI for measurement of $\mathrm{PSD}_{\text {muscle }}$ was placed in muscle tissue medially adjacent to the sciatic nerve (M. semimembranosus or adductor magnus). After slice-wise calculation of T2 and PSD, parameters were averaged from all six slices for further analysis.

Table 2 Descriptive statistics of multiparametric sciatic nerve MRN

\begin{tabular}{|c|c|c|c|c|c|c|}
\hline & \multicolumn{2}{|l|}{ Scan 1} & \multicolumn{2}{|l|}{ Scan 2} & \multicolumn{2}{|l|}{ Scan 3} \\
\hline & Reader 1 & Reader 2 & Reader 1 & Reader 2 & Reader 1 & Reader 2 \\
\hline MTR (\%) & $26.8 \pm 4.2$ & $26.84 \pm 4.2$ & $26.97 \pm 3.0$ & $27.03 \pm 3.0$ & $26.35 \pm 3.4$ & $26.52 \pm 3.3$ \\
\hline $\mathrm{T} 2(\mathrm{~ms})$ & $63.93 \pm 8.7$ & $64.28 \pm 8.6$ & $65.57 \pm 8.4$ & $65.72 \pm 8.3$ & $63.66 \pm 8.1$ & $64.1 \pm 8.1$ \\
\hline PSD & $344.3 \pm 78.9$ & $334.7 \pm 82.7$ & $341.7 \pm 74.2$ & $331.6 \pm 76.5$ & $351.9 \pm 84.2$ & $341.4 \pm 84$ \\
\hline Normalized PSD & $0.7 \pm 0.09$ & $0.69 \pm 0.1$ & $0.71 \pm 0.09$ & $0.71 \pm 0.1$ & $0.71 \pm 0.09$ & $0.71 \pm 0.09$ \\
\hline $\mathrm{CSA}\left(\mathrm{mm}^{2}\right)$ & $18.1 \pm 4.5$ & $18.9 \pm 4.4$ & $18.4 \pm 4.6$ & $19.1 \pm 4.6$ & $18.4 \pm 4.6$ & $18.7 \pm 4.9$ \\
\hline
\end{tabular}

All values represent mean \pm standard deviation. MTR magnetization transfer ratio, $T 2$ transverse relaxation time, $P S D$ proton spin density, CSA crosssectional area 

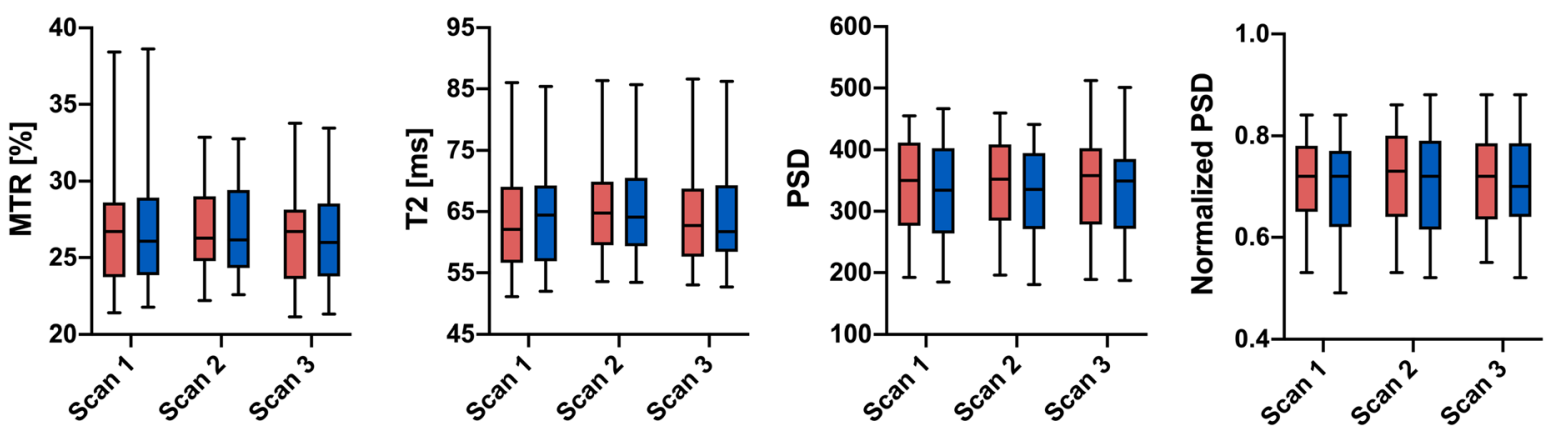

Fig. 3 Descriptive statistics for both readers and all three scans. Values are illustrated as boxplots with whiskers to visualize measurement distribution (box showing the 25 th to 75 th percentiles with a line at the

\section{Magnetization transfer ratio}

MTR was calculated based on sciatic nerve signal intensity in the pair of GRE sequences without $\left(\mathrm{S}_{0}\right)$ and with $\left(\mathrm{S}_{1}\right)$ offresonance saturation pulse using the equation:

$\operatorname{MTR}=100 \times \frac{\left(S_{0}-S_{1}\right)}{S_{0}}$

Analogously to T2 relaxometry, segmentation information was derived from the high-resolution T2 sequence. Likewise, signal intensity was averaged over all voxels to reduce possible effects of noise. If necessary, ROIs were manually adjusted for distortion or motion artifacts by both readers independently using the images without the off-resonance pulse. Additional computational co-registration between the two MTI sequences was not applied [37], as we were able to ascertain visually that ROIs aligned well with the nerve contours in both sequences (Fig. 2B). Subsequently, MTR was calculated for every slice and then averaged over all six slices.

\section{Statistical analysis}

All values are shown as mean \pm standard deviation. $p$ values $\leq$ 0.05 were regarded as statistically significant. Statistical median, whiskers indicate the minimum and maximum data values). $\mathrm{MTR}=$ magnetization transfer ratio, $\mathrm{T} 2=$ transverse relaxation time, $\mathrm{PSD}=$ proton spin density

analyses were performed using SPSS (Version 24; SPSS Inc.) or R (Version 4.0.3; R Foundation for Statistical Computing). Graphs were created using GraphPad Prism (Version 8.3; GraphPad Software Inc.).

A single measurement, absolute agreement, two-way random effects model, ICC $(2,1)$ according to Shrout and Fleiss was applied to assess interreader agreement and to calculate ICCs with 95\% confidence intervals (CIs). To estimate test-retest reliability, a single measurement, absolute agreement, two-way mixed effects model was applied to calculate ICC $(3,1)$ for each observer separately, and CIs were calculated accordingly. ICC values between 0.5 and 0.75 , between 0.75 and 0.9 , and greater 0.9 were regarded as moderate, good, and excellent agreement [30]. In addition, a Bland-Altmann analysis for repeated measurements was conducted and illustrated in Bland-Altmann plots. To assess measurement accuracy between readers and scans, SEM was calculated according to Popovic and Thomas [31]. MDD values for a $\mathrm{CI}$ of $95 \%$ were calculated according to the formula MDD $=\operatorname{SEM} \times 1.96 \times \sqrt{ } 2[31,32]$.

\section{Results}

For the sciatic nerve, overall mean \pm standard deviation MTR was $26.75 \pm 3.5 \%$, mean T2 relaxation time was $64.54 \pm 8.2$

Table 3 Intraclass correlation coefficients (ICCs), standard error of measurement (SEM), and minimum detectable difference (MDD) values for interreader reliability and test-retest reliability

\begin{tabular}{|c|c|c|c|c|c|c|c|}
\hline & \multirow[t]{2}{*}{ Interreader ICC } & \multicolumn{2}{|l|}{ Test-retest ICC } & \multicolumn{2}{|l|}{ SEM } & \multicolumn{2}{|l|}{ MDD } \\
\hline & & Reader 1 & Reader 2 & Interreader & $\begin{array}{l}\text { Test- } \\
\text { retest }\end{array}$ & Interreader & $\begin{array}{l}\text { Test- } \\
\text { retest }\end{array}$ \\
\hline MTR & $0.81(0.60-0.92)$ & $0.79(0.62-0.90)$ & $0.75(0.55-0.88)$ & $1.7 \%$ & $1.7 \%$ & $4.7 \%$ & $4.7 \%$ \\
\hline $\mathrm{T} 2$ & $0.92(0.81-0.97)$ & $0.92(0.84-0.96)$ & $0.9(0.80-0.95)$ & $2.67 \mathrm{~ms}$ & $2.66 \mathrm{~ms}$ & $7.4 \mathrm{~ms}$ & $7.37 \mathrm{~ms}$ \\
\hline PSD & $0.94(0.85-0.98)$ & $0.94(0.87-0.97)$ & $0.94(0.88-0.97)$ & 21.3 & 20.1 & 59 & 55.7 \\
\hline Normalized PSD & $0.89(0.74-0.95)$ & $0.9(0.81-0.96)$ & $0.89(0.78-0.95)$ & 0.03 & 0.03 & 0.08 & 0.08 \\
\hline
\end{tabular}

Intraclass correlation coefficients (ICCs) with 95\% confidence-interval were calculated according to Shrout and Fleiss. SEM and MDD calculations were based on Popovic and Thomas [31]. MTR magnetization transfer ratio, T2 transverse relaxation time, PSD proton spin density 

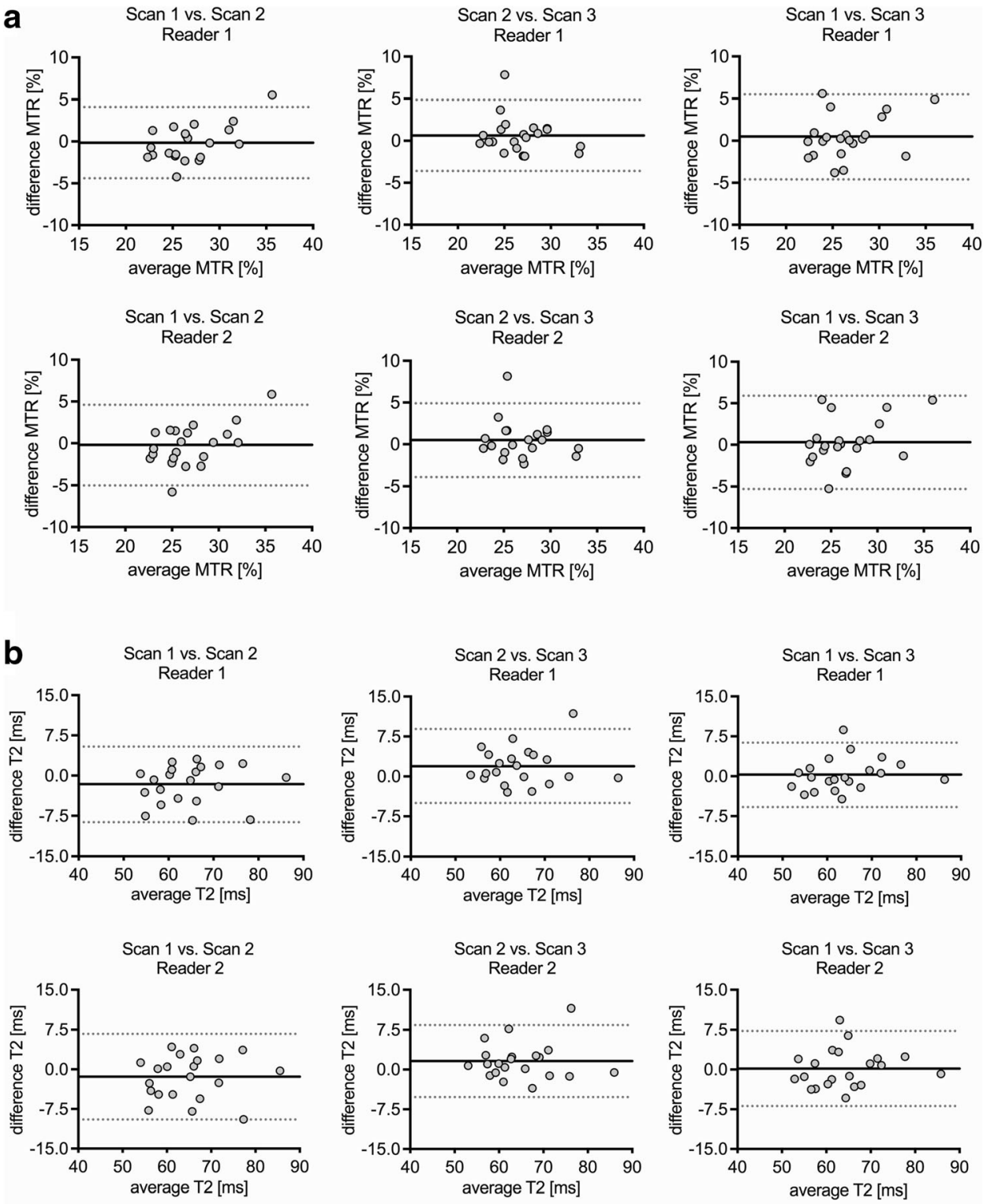

C

Scan 1 vs. Scan 2
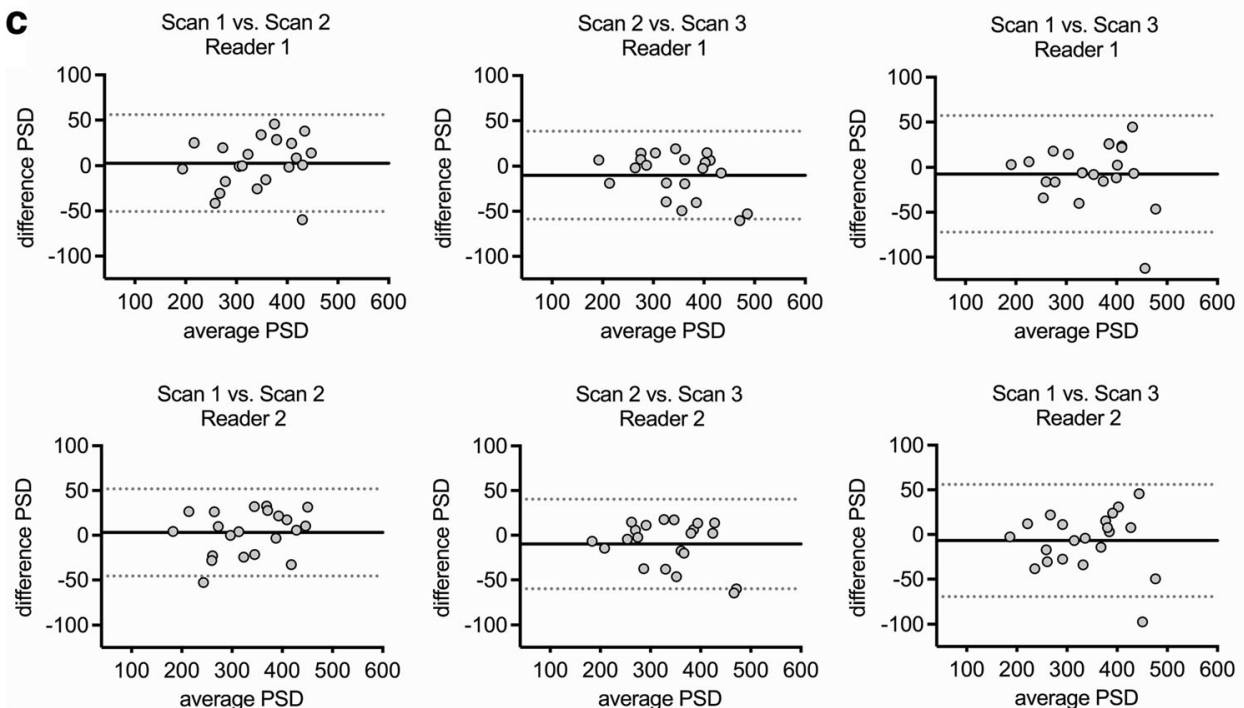
Fig. 4 Bland-Altman plots for assessment of test-retest reliability of magnetization transfer ratio (MTR) (a), transverse relaxation time T2 (b), and proton spin density (PSD) (c) for both observers and all three scans, respectively. The black continuous line represents the mean of all differences (bias), grey dotted lines show the $95 \%$ limits of agreement

ms, and mean PSD was $340.93 \pm 78.8$. Overall normalized PSD was $0.71 \pm 0.09$. Detailed descriptive statistics for every parameter, all three scans, and both readers are presented in Table 2 and Fig. 3.

Assessment of interreader reliability overall resulted in ICC values between 0.81 (for MTR) and 0.94 (for PSD), implying good to excellent agreement according to Koo and $\mathrm{Li}$ [30]. Calculation of test-retest reliability resulted in slightly lower ICCs ranging from 0.75 to 0.94 . Lowest ICCs were observed for nerve MTR and highest for nerve PSD, respectively.

SEM for MTR was $1.7 \%$ for both interreader and test-retest agreement, SEM for T2 was $2.67 \mathrm{~ms}$ for interreader and $2.66 \mathrm{~ms}$ for test-retest agreement, and SEM for PSD was 21.3 and 20.1 for interreader and test-retest agreement, respectively. ICC, SEM, and MDD values for all parameters are listed in Table 3.

Bland-Altmann plots for test-retest reliability are shown in Fig. 4 and for interreader reliability in Fig. 5. Bland-Altman analysis showed a rather random distribution of measurement error with low bias between raters and scans. Also, no proportional bias such as systematically higher measurement error for higher or lower MTR, T2, or PSD values could be observed. Overall mean differences between readers were $0.1 \%$ for MTR, $0.3 \mathrm{~ms}$ for T2, and 10.1 for PSD.

\section{Discussion}

This study evaluates the interreader and test-retest reliability of quantitative MRN biomarkers like MTR, T2 relaxation time, and PSD of the sciatic nerve in a cohort of 21 healthy participants. Each participant underwent three MRN exams on separate days which were analyzed by two independent readers. By reporting ICCs, SEM, and MDD values, we quantify measurement imprecision of all readout parameters.
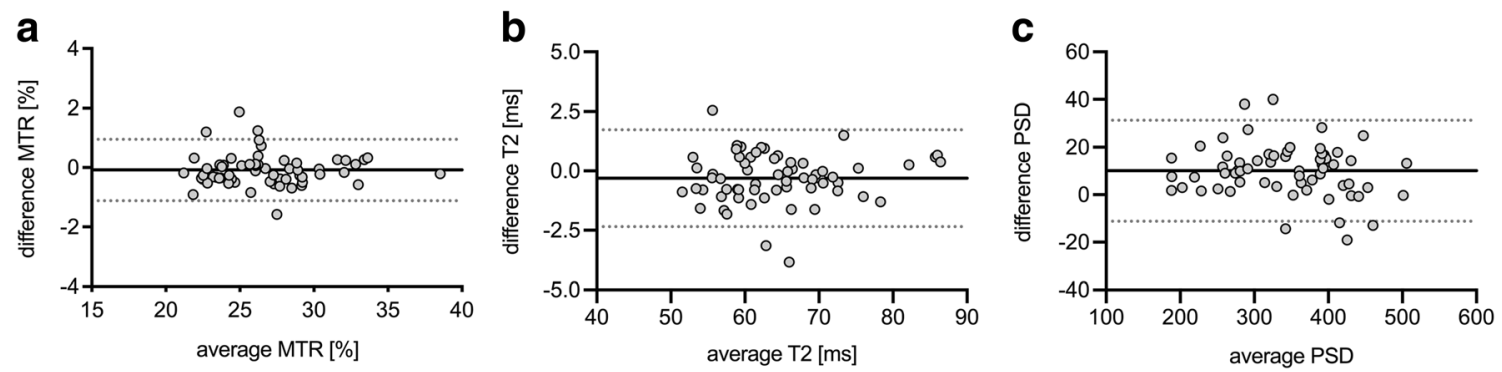

Fig. 5 Bland-Altman plots for assessment of interreader reliability of magnetization transfer ratio (MTR) (a), transverse relaxation time T2 (b), and proton spin density (PSD) (c) of the sciatic nerve. The black
While T2 relaxometry and MTI are increasingly studied as quantitative MRN techniques, they have not been implemented in clinical routine yet [11-14, 24, 26]. Before interpreting MRN biomarkers in individual patients, assessment of reliability is required not only in qualitative categories but particularly by precise quantification of measurement error.

This study adds to the field by providing a systematic quantification of interreader and test-retest reliability in a larger cohort for both peripheral nerve MTI and T2 relaxometry biomarkers. With regard to peripheral nerve MTI, we are aware of two previous studies that have assessed reliability in smaller cohorts (Yiannakas et al [28]: 5 participants, $\mathrm{ICC}_{\text {interreader }} 0.65, \mathrm{ICC}_{\text {test-retest }}$ 0.76; Dortch et al [11]: 13 participants, $\mathrm{ICC}_{\text {interreader }} 0.92, \mathrm{ICC}_{\text {test-retest }} 0.69$ ), although the main focus of these studies was on other research concerns $[11,28]$. Regarding T2 relaxometry, Sollmann et al [29] have recently reported promising first results for interreader reliability while test-retest reliability had not been assessed yet to our knowledge.

While the ICC is a widely used index of reliability, it depends not only on the measurement error itself but also on the variance of the measured parameter in the examined cohort [38-40]. To provide a parameter of measurement imprecision that is independent of the variance of the biomarker in the test cohort, we here additionally report the SEM for all assessed biomarkers.

Moreover, the SEM allows for the calculation of the MDD, which indicates the difference in repeated measurements needed to attribute a measured difference to a change in the true value with a $95 \%$ certainty, but not to a fluctuation due to measurement error $[31,32]$. The SEM and MDD are therefore of high significance when implementing quantitative biomarkers in clinical practice.

To value the clinical relevance of the measurement error of $\mathrm{T} 2$ relaxometry and MTI, the MDD can be regarded in context with results from previous studies in the setting of specific neuropathies and healthy controls as summarized in Tables 4 and 5.

For MTR of the sciatic nerve, the current study implies an MDD value of $4.7 \%$. In previous studies, significant differences in MTR between patients and healthy controls ranged continuous line represents the mean of all differences (bias), grey dotted lines show the $95 \%$ limits of agreement 
Table 4 MTR values in health and disease as reported in previous studies of peripheral nerve imaging (non-exhaustive)

\begin{tabular}{|c|c|c|c|c|c|}
\hline Authors & Examined pathology & Examined nerve & $\begin{array}{l}\text { Healthy controls: } \\
{[\text { mean } \mathrm{MTR} \pm \mathrm{SD} \text { in }} \\
\%]\end{array}$ & $\begin{array}{l}\text { Disease: } \\
{[\text { mean } \mathrm{MTR} \pm \mathrm{SD} \text { in }} \\
\%]\end{array}$ & $\begin{array}{l}\text { Difference mean } \\
\text { MTR in } \% \\
\text { (healthy - disease) }\end{array}$ \\
\hline Dortch et al [11] & $\begin{array}{l}\text { Charcot-Marie-Tooth types } \\
1 \mathrm{~A} \text { and } 2 \mathrm{~A}\end{array}$ & Sciatic nerve & $37.2 \pm 2.3(\mathrm{n}=21)$ & $\begin{array}{l}33.8 \pm 3.3 \text { and } \\
31.5 \pm 1.9 \\
(\mathrm{n}=10 \text { and } 3)\end{array}$ & $\frac{3.4(1 \mathrm{~A})}{\underline{(2 \mathrm{~A})}}$ and $\underline{5.7}$ \\
\hline $\begin{array}{l}\text { Fortanier et al } \\
\quad[12]\end{array}$ & Charcot-Marie-Tooth types 1A & $\begin{array}{l}\text { Sciatic and tibial } \\
\text { nerve }\end{array}$ & $39.5 \pm 3^{\dagger}(\mathrm{n}=13)$ & $33.7 \pm 3.7^{\dagger}(\mathrm{n}=32)^{\dagger}$ & $\underline{5.8}$ \\
\hline $\begin{array}{l}\text { Kollmer et al } \\
\text { [14] }\end{array}$ & Hereditary transthyretin amyloidosis & Sciatic nerve & $39.4 \pm 2.1(\mathrm{n}=20)$ & $26.4 \pm 0.7^{\star}(\mathrm{n}=25)^{\ddagger}$ & $\underline{13}$ \\
\hline $\begin{array}{l}\text { Kollmer et al } \\
{[24]}\end{array}$ & $\begin{array}{l}\text { 5q-linked spinal muscular atrophy } \\
\text { (SMA 3) }\end{array}$ & Sciatic nerve & $32.4 \pm 0.6(\mathrm{n}=18)$ & $26.2 \pm 0.7(\mathrm{n}=18)$ & $\underline{6.2}$ \\
\hline $\begin{array}{l}\text { Kollmer et al } \\
{[41]}\end{array}$ & $\begin{array}{l}\text { Dependency of MTI on age and } \\
\text { location, only healthy participants }\end{array}$ & $\begin{array}{l}\text { Sciatic and tibial } \\
\text { nerve }\end{array}$ & $19.9 \pm 1^{*}(\mathrm{n}=10)$ & - & - \\
\hline $\begin{array}{l}\text { Pridmore et al } \\
\text { [42] }\end{array}$ & $\begin{array}{l}\text { Hereditary neuropathy with liability } \\
\text { to pressure palsies }\end{array}$ & Sciatic nerve & $39.4 \pm 2(n=7)$ & $37.6 \pm 3.8(\mathrm{n}=10)$ & 1.8 \\
\hline
\end{tabular}

Mean values for magnetization transfer ratio (MTR) as described by previous studies for various diseases. ${ }^{\dagger}$ Only sciatic nerve values are displayed, ${ }^{*}$ values for patients with symptomatic polyneuropathy, ${ }^{*}$ values for the distal sciatic nerve in a younger age group (20-32 years). Underlined differences were considered significant. Measures of precision as the standard error of measurement (SEM) and the minimum detectable difference (MDD) may be interpreted in conjunction with these values

from 3.4 to $13 \%$, and in three out of four studies a difference of over $4.7 \%$ was observed (Table 4) [11, 12, 14, 24]. Notably, the mean MTR of our cohort was lower than in most of the control groups listed in Table 4. This most likely has technical reasons, since MTR values depend on various factors, such as magnetic field strength, coil characteristics, and sequence parameters, like offset frequency and power of the saturation pulses [47-50].

For T2 of the sciatic nerve, the present study implies an MDD of approximately $7.4 \mathrm{~ms}$. For comparison, previously reported significant differences of $\mathrm{T} 2$ ranged from 10.8 to $26.6 \mathrm{~ms}$ between patients and control groups (Table 5) [13, 25, 29, 43, 44].

For PSD, our results imply an MDD of 55.7 (test-retest) and 59 (interreader). PSD is considered a semi-quantitative parameter that directly depends on the MR signal and associated parameters, such as receiver gain. Therefore, reliability values of PSD reported in this study may only be regarded as orientation values and in conjunction with the absolute mean values of other studies (Table 5). To overcome this limitation, we additionally normalized PSD values by using adjacent muscles. Although these normalized PSD values may serve as a more robust parameter when using different setups, they may however be influenced by muscular changes which can occur in the context of systemic neuropathies [51].

While the calculated MDDs are often smaller than the mean differences between patients and healthy controls, it is not clear whether changes in biomarkers in longitudinal follow-up studies will exceed these limits. So far, we are not aware of any longitudinal studies assessing MTR, T2, or PSD in the PNS. However, such longitudinal studies are crucial to further evaluate the potential of quantitative MRN in a clinical follow-up setting.
Although quantitative MRN offers multiple contrasts, neuropathies of different etiologies share non-specific changes as an increase in T2 or PSD and a decrease in MTR, which may limit their potential in differential diagnosis [52]. Furthermore, normative data may depend on scanning parameters, demography, and postprocessing methods [33, 41]. However, if reliability is proven, quantitative biomarkers could allow tracking disease progression or responsiveness under therapy. In order to implement quantitative MRN as a clinical tool, more studies on normative data should be carried out in the future.

Our study has limitations. First, we restricted the analysis to the sciatic nerve, since it is the most commonly examined nerve and well suited for MRN due to its large caliber and straight course and therefore most appropriate for MSE and GRE sequences with lower special resolution. An inclusion of smaller nerves or nerves with an oblique course may have led to different values of measurement error. Thus, all values presented should primarily be used for MRN of the sciatic nerve and may generally serve as orientation values under optimal conditions. Second, our cohort only consisted of healthy young participants and demographic variables were relatively homogeneous. This also allowed for assessment of measurement error under rather good conditions, since from our experience motion artifacts are less commonly observed in younger subjects. The examined cohort was male, however, we would not expect different results for female participants, since all parameters have been shown to not systematically differ between sexes [33, 41]. Furthermore, planning of the MR examination and image postprocessing were performed by experienced neuromuscular radiologists, and also for this reason the proposed orientational values should rather be 
Table 5 Nerve transverse relaxation times T2 and/or proton spin density (PSD) values in various diseases as reported in previous studies of peripheral nerve imaging (non-exhaustive)

\begin{tabular}{|c|c|c|c|c|c|}
\hline Authors & Examined pathology & Examined nerve & $\begin{array}{l}\text { Healthy controls: } \\
{[\text { mean } \pm \mathrm{SD}, \mathrm{T} 2 \text { in } \mathrm{ms}]}\end{array}$ & $\begin{array}{l}\text { Disease: [mean } \pm \\
\text { SD, T2 in ms] }\end{array}$ & $\begin{array}{l}\text { Difference } \\
\text { mean } \\
\text { T2/PSD } \\
\text { (healthy - disease) }\end{array}$ \\
\hline Vaeggemose et al [4] & $\begin{array}{l}\text { Type } 1 \text { diabetes with } \\
\text { diabetic neuropathy }\end{array}$ & Sciatic nerve & $\begin{array}{l}\text { T2: } 79 \pm 8 \\
\text { PSD: } 381 \pm 80 \\
(n=30)\end{array}$ & $\begin{array}{l}\text { T2: } 83 \pm 7^{*} \\
\text { PSD: } 343 \pm 77^{*} \\
(\mathrm{n}=11)^{*}\end{array}$ & $\begin{array}{l}4 \\
38\end{array}$ \\
\hline Fortanier et al [12] & Charcot-Marie-Tooth types 1A & Sciatic and tibial nerve & $\begin{array}{l}\text { PSD: } 490 \pm 65 \\
(\mathrm{n}=13)\end{array}$ & $\begin{array}{l}\text { PSD: } 557 \pm 89^{\dagger} \\
(\mathrm{n}=32)\end{array}$ & $\underline{67}$ \\
\hline Kollmer et al [13] & $\begin{array}{l}\text { Amyloid light chain } \\
\text { (AL) amyloidosis }\end{array}$ & $\begin{array}{l}\text { Sciatic nerve and distal } \\
\text { branches }\end{array}$ & $\begin{array}{l}\text { T2: } 75.4 \pm 2 \\
\text { PSD: } 310.5 \pm 14.1 \\
(n=25)\end{array}$ & $\begin{array}{l}\text { T2: } 102 \pm 14.4^{+} \\
\text {PSD: } 525.5 \pm 53^{+} \\
(n=7)^{+}\end{array}$ & $\frac{26.6}{215}$ \\
\hline Jende et al [25] & Multiple sclerosis & $\begin{array}{l}\text { Sciatic nerve and distal } \\
\text { branches }\end{array}$ & $\begin{array}{l}\text { T2: } 82 \pm 2.1 \\
\text { PSD: } 266 \pm 11 \\
(n=35)\end{array}$ & $\begin{array}{l}\text { T2: } 64.3 \pm 1 \\
\text { PSD: } 371.8 \pm 7.7 \\
(n=36)\end{array}$ & $\frac{17.7}{105.8}$ \\
\hline Kronlage et al [26] & $\begin{array}{l}\text { Chronic inflammatory } \\
\text { demyelinating polyneuropathy }\end{array}$ & Sciatic nerve & $\begin{array}{l}\text { T2: } 70.3 \pm 1.4 \\
\text { PSD: } 159.7 \pm 7.1 \\
(n=18)\end{array}$ & $\begin{array}{l}\text { T2: } 72.3 \pm 2.9 \\
\text { PSD: } 177.2 \pm 7.4 \\
(n=18)\end{array}$ & $\begin{array}{l}2 \\
17.5 \\
\end{array}$ \\
\hline Vaeggemose et al [27] & Charcot-Marie-Tooth types $1 \mathrm{~A}$ & Sciatic and tibial nerve & $\begin{array}{l}\text { T2: } 79 \\
\text { PSD: } 403 \\
(n=30)\end{array}$ & $\begin{array}{l}\text { T2: } 81 \\
\text { PSD: } 561 \\
(\mathrm{n}=15)\end{array}$ & $\begin{array}{l}2 \\
158 \\
\end{array}$ \\
\hline Sollmann et al [29] & $\begin{array}{l}\text { Lumbar disc herniation } \\
\text { with nerve compression }\end{array}$ & Sciatic nerve & $\begin{array}{l}\mathrm{T} 2: 43.3 \pm 2.4 \\
(\mathrm{n}=21)\end{array}$ & $\begin{array}{l}\text { T2: } 61.5 \pm 6.2^{*} \\
(\mathrm{n}=5)\end{array}$ & $\underline{18.2}$ \\
\hline Kollmer et al [43] & $\begin{array}{l}\text { Transthyretin familial } \\
\text { amyloid polyneuropathy } \\
\text { (TTR-FAP) }\end{array}$ & Sural nerve & $\begin{array}{l}\text { T2: } 79 \pm 2.7 \\
\text { PSD: } 258.2 \pm 9.1 \\
(n=35)\end{array}$ & $\begin{array}{l}\text { T2: } 89.4 \pm 5.1^{\S} \\
\text { PSD: } 430 \pm 15.3^{\S} \\
(\mathrm{n}=25)^{\S}\end{array}$ & $\frac{10.4}{171.4}$ \\
\hline Kollmer et al [44] & $\begin{array}{l}\text { Transthyretin familial } \\
\text { amyloid polyneuropathy } \\
\text { (TTR-FAP) }\end{array}$ & $\begin{array}{l}\text { Sciatic nerve and distal } \\
\text { branches }\end{array}$ & $\begin{array}{l}\text { T2: } 84.1 \pm 2.5 \\
\text { PSD: } 286.6 \pm 10 \\
(\mathrm{n}=40)\end{array}$ & $\begin{array}{l}\text { T2: } 103.9 \pm 6.4^{\S} \\
\text { PSD: } 550 \pm 35.8^{\S} \\
(\mathrm{n}=13)^{\S}\end{array}$ & $\frac{19.8}{\underline{263.4}}$ \\
\hline Felisaz et al [45] & $\begin{array}{l}\text { Chronic inflammatory } \\
\text { demyelinating } \\
\text { polyneuropathy }\end{array}$ & Tibial nerve & $\begin{array}{l}\text { T2: } 55.7 \\
\text { (range: } 30-94) \\
(n=10)\end{array}$ & $\begin{array}{l}\text { T2: } 52.8 \\
\text { (range: } 32-81) \\
(\mathrm{n}=10)\end{array}$ & 2.9 \\
\hline Pham et al [46] & Diabetic neuropathy & $\begin{array}{r}\text { Sciatic nerve and } \\
\text { distal branches }\end{array}$ & $\begin{array}{l}\text { PSD: } 288 \pm 13.4 \\
(\mathrm{n}=25)\end{array}$ & $\begin{array}{l}\text { PSD: } 360 \pm 22.9^{\#} \\
(\mathrm{n}=10)^{\#}\end{array}$ & $\underline{72}$ \\
\hline
\end{tabular}

Mean values for T2 and proton spin density (PSD) as described by previous studies for various diseases. ${ }^{*}$ Values for patients with severe polyneuropathy, ${ }^{\dagger}$ only sciatic nerve values are displayed, ${ }^{+}$values for patients with moderate AL-polyneuropathy, ${ }^{\dagger}$ acquired using a T2-prepared turbo spin-echo sequence, ${ }^{\S}$ values for patients with symptomatic TTR-FAP, ${ }^{\#}$ values for patients with severe polyneuropathy. Underlined differences were considered significant. Measures of precision as the standard error of measurement (SEM) and the minimum detectable difference (MDD) may be interpreted in conjunction with these values

regarded as minimum values. Besides, MTR may depend on B1 field inhomogeneities, which is why correction schemes may be applied to neuromuscular imaging $[11,12,53]$. To reduce the effects of $\mathrm{B} 1$ field inhomogeneities on the received signal, we applied an adaptive inline image filter and restricted analysis to six central slices where the B1 field is expected to be more homogeneous than at the edges of the slab. Lastly, this is a single-center, single-vendor study and measured parameters may depend on the hardware, sequence parameters, and postprocessing methods, limitations that are inherent to most quantitative imaging studies. While we measured testretest reliability in a follow-up setting with one particular scanner, we would expect higher measurement error if multiple scanners were used, as differences in amplification of the MR signal modify PSD and differences in saturation pulses may affect MTR.
In conclusion, this study demonstrates that MTI and T2 relaxometry of the sciatic nerve results in reliable and reproducible values. By assessing the SEM for all examined parameters, it provides quantitative data to measure the imprecision that is associated with multiple scans or different observers. These values may be considered as orientation values of measurement error in further studies and potential clinical applications of quantitative MRN.

Supplementary Information The online version contains supplementary material available at https://doi.org/10.1007/s00330-021-08072-9.

Funding Open Access funding enabled and organized by Projekt DEAL. Prof. Heiland (SFB 1118), Dr. Schwarz (SFB 1118), and Prof. Bendszus (SFB 1158) were supported by the German Research Foundation. Dr. 
Preisner and Dr. Schwarz were supported by a Physician-Scientist Fellowship of the Medical Faculty of the University of Heidelberg. Dr. Foesleitner receives a research grant from the Rahel Goitein-Straus Support Program of the Medical Faculty of the University of Heidelberg. For the remaining authors, none were declared.

\section{Declarations}

Guarantor The scientific guarantor of this publication is Moritz Kronlage.

Conflict of Interest The authors of this manuscript declare no relationships with any companies whose products or services may be related to the subject matter of the article.

Statistics and Biometry Rouven Behnisch (Institute of Medical Biometry and Informatics, University of Heidelberg) conducted the statistical analysis and is co-author of this manuscript.

Informed Consent Written informed consent was obtained from all subjects (patients) in this study.

Ethical Approval Institutional Review Board approval was obtained.

Study subjects or cohorts overlap Several subjects of this study also participated in another study on diffusion tensor imaging (DTI): Preisner F, Bäumer P, Wehrstein M et al (2019) Peripheral Nerve Diffusion Tensor Imaging : Interreader and Test-retest Reliability as Quantified by the Standard Error of Measurement. Clin Neuroradiol. 10.1007/ s00062-019-00859-0.

Apart from the demographic variables, there is no overlap especially not in results or conclusions as all data concerning MTI and T2Relaxometry have not been analyzed before.

\section{Methodology}

- prospective study

- observational

- performed at one institution

Open Access This article is licensed under a Creative Commons Attribution 4.0 International License, which permits use, sharing, adaptation, distribution and reproduction in any medium or format, as long as you give appropriate credit to the original author(s) and the source, provide a link to the Creative Commons licence, and indicate if changes were made. The images or other third party material in this article are included in the article's Creative Commons licence, unless indicated otherwise in a credit line to the material. If material is not included in the article's Creative Commons licence and your intended use is not permitted by statutory regulation or exceeds the permitted use, you will need to obtain permission directly from the copyright holder. To view a copy of this licence, visit http://creativecommons.org/licenses/by/4.0/.

\section{References}

1. Chhabra A, Madhuranthakam AJ, Andreisek G (2018) Magnetic resonance neurography: current perspectives and literature review. Eur Radiol 28:698-707

2. Kronlage M, Knop KC, Schwarz D et al (2019) Amyotrophic lateral sclerosis versus multifocal motor neuropathy: utility of MR neurography. Radiology 292:149-156
3. Hilgenfeld T, Jende J, Schwarz D et al (2017) Somatotopic fascicular lesions of the brachial plexus demonstrated by high-resolution magnetic resonance neurography. Invest Radiol 52:741-746

4. Vaeggemose M, Pham M, Ringgaard S et al (2017) Magnetic resonance neurography visualizes abnormalities in sciatic and tibial nerves in patients with type 1 diabetes and neuropathy. Diabetes 66:1779-1788

5. Preisner F, Bendszus M, Schwarz D (2020) Visualization of direct median nerve damage following transbrachial arterial access. JACC Cardiovasc Interv 13:1265-1266

6. Upadhyaya V, Upadhyaya DN, Mishra B (2018) MR neurography in traumatic, non-obstetric paediatric brachial plexopathy. Eur Radiol 28:2417-2424

7. Martín Noguerol T, Barousse R, Gómez Cabrera M, Socolovsky M, Bencardino JT, Luna A (2019) Functional MR neurography in evaluation of peripheral nerve trauma and postsurgical assessment. Radiographics 39:427-446

8. Kronlage M, Pitarokoili K, Schwarz D et al (2017) Diffusion tensor imaging in chronic inflammatory demyelinating polyneuropathy: diagnostic accuracy and correlation with electrophysiology. Invest Radiol 52:701-707

9. Vaeggemose M, Pham M, Ringgaard S et al (2017) Diffusion tensor imaging MR neurography for the detection of polyneuropathy in type 1 diabetes. J Magn Reson Imaging 45:1125-1134

10. Mazal AT, Ashikyan O, Cheng J, Le LQ, Chhabra A (2019) Diffusion-weighted imaging and diffusion tensor imaging as adjuncts to conventional MRI for the diagnosis and management of peripheral nerve sheath tumors: current perspectives and future directions. Eur Radiol 29:4123-4132

11. Dortch RD, Dethrage LM, Gore JC, Smith SA, Li J (2014) Proximal nerve magnetization transfer MRI relates to disability in Charcot-Marie-Tooth diseases. Neurology 83:1545-1553

12. Fortanier E, Ogier AC, Delmont E et al (2020) Quantitative assessment of sciatic nerve changes in Charcot-Marie-Tooth type 1A patients using magnetic resonance neurography. Eur J Neurol 27: 1382-1389

13. Kollmer J, Weiler M, Purrucker J et al (2018) MR neurography biomarkers to characterize peripheral neuropathy in AL amyloidosis. Neurology 91:e625-e634

14. Kollmer J, Hegenbart U, Kimmich C et al (2020) Magnetization transfer ratio quantifies polyneuropathy in hereditary transthyretin amyloidosis. Ann Clin Transl Neurol 7:799-807

15. Boulby PA (2003) T2: the transverse relaxation time. In: Tofts PS (ed) Quantitative MRI of the Brain: Measuring Changes Caused by Disease. Wiley, pp 143-201

16. Tofts PS, du Boulay EP (1990) Towards quantitative measurements of relaxation times and other parameters in the brain. Neuroradiology 32:407-415

17. Tofts PS (2003) Proton density of tissue water. In: Tofts PS (ed) Quantitative MRI of the Brain: Measuring Changes Caused by Disease. Wiley, pp 83-108

18. MacKay A, Whittall K, Adler J, Li D, Paty D, Graeb D (1994) In vivo visualization of myelin water in brain by magnetic resonance. Magn Reson Med 31:673-677

19. Wolff SD, Balaban RS (1989) Magnetization transfer contrast (MTC) and tissue water proton relaxation in vivo. Magn Reson Med 10:135-144

20. Does MD, Beaulieu C, Allen PS, Snyder RE (1998) Multicomponent $\mathrm{T} 1$ relaxation and magnetisation transfer in peripheral nerve. Magn Reson Imaging 16:1033-1041

21. Grossman RI, Gomori JM, Ramer KN, Lexa FJ, Schnall MD (1994) Magnetization transfer: theory and clinical applications in neuroradiology. Radiographics 14:279-290

22. Moccia M, van de Pavert S, Eshaghi A et al (2020) Pathological correlates of the magnetization transfer ratio in multiple sclerosis. Neurology. https://doi.org/10.1212/wnl.0000000000010909 
23. Odrobina EE, Lam TY, Pun T, Midha R, Stanisz GJ (2005) MR properties of excised neural tissue following experimentally induced demyelination. NMR Biomed 18:277-284

24. Kollmer J, Kessler T, Sam G et al (2020) Magnetization transfer ratio: a quantitative imaging biomarker for $5 \mathrm{q}$ spinal muscular atrophy. Eur J Neurol. https://doi.org/10.1111/ene.14528

25. Jende JME, Hauck GH, Diem R et al (2017) Peripheral nerve involvement in multiple sclerosis: demonstration by magnetic resonance neurography. Ann Neurol 82:676-685

26. Kronlage M, Baumer P, Pitarokoili K et al (2017) Large coverage MR neurography in CIDP: diagnostic accuracy and electrophysiological correlation. J Neurol 264:1434-1443

27. Vaeggemose M, Vaeth S, Pham M et al (2017) Magnetic resonance neurography and diffusion tensor imaging of the peripheral nerves in patients with Charcot-Marie-Tooth Type 1A. Muscle Nerve 56: E78-E84

28. Yiannakas MC, Schneider T, Yoneyama M et al (2020) Magnetisation transfer ratio combined with magnetic resonance neurography is feasible in the proximal lumbar plexus using healthy volunteers at 3T. Sci Rep 10:14568

29. Sollmann N, Weidlich D, Klupp E et al (2020) T2 mapping of the distal sciatic nerve in healthy subjects and patients suffering from lumbar disc herniation with nerve compression. MAGMA 33:713724

30. Koo TK, Li MY (2016) A guideline of selecting and reporting intraclass correlation coefficients for reliability research. J Chiropr Med 15:155-163

31. Popovic ZB, Thomas JD (2017) Assessing observer variability: a user's guide. Cardiovasc Diagn Ther 7:317-324

32. Weir JP (2005) Quantifying test-retest reliability using the intraclass correlation coefficient and the SEM. J Strength Cond Res 19:231240

33. Kronlage M, Schwehr V, Schwarz D et al (2019) Magnetic resonance neurography : normal values and demographic determinants of nerve caliber and T2 relaxometry in 60 healthy individuals. Clin Neuroradiol 29:19-26

34. Milford D, Rosbach N, Bendszus M, Heiland S (2015) Monoexponential fitting in T2-relaxometry: relevance of offset and first echo. PLoS One 10:e0145255

35. Thorpe JW, Barker GJ, Jones SJ et al (1995) Magnetisation transfer ratios and transverse magnetisation decay curves in optic neuritis: correlation with clinical findings and electrophysiology. J Neurol Neurosurg Psychiatry 59:487-492

36. Tofts PS (2003) T2: the transverse relaxation time. In: Tofts PS (ed) Quantitative MRI of the Brain: Measuring Changes Caused by Disease. Wiley, pp 143-201

37. Nagy SA, Váczi M, Perlaki G, Orsi G, Illés Z, Bogner P (2019) Acute and delayed effects of concentric and eccentric exercise on quantitative muscle MRI. Acta Radiol 60:902-907

38. de Vet HC, Terwee CB, Knol DL, Bouter LM (2006) When to use agreement versus reliability measures. J Clin Epidemiol 59:10331039
39. Ten Cate DF, Luime JJ, Hazes JM, Jacobs JW, Landewé R (2010) Does the intraclass correlation coefficient always reliably express reliability? Comment on the article by Cheung et al. Arthritis Care Res (Hoboken) 62:1357-1358 author reply 1358

40. Preisner F, Bäumer P, Wehrstein M et al (2019) Peripheral nerve diffusion tensor imaging : interreader and test-retest reliability as quantified by the standard error of measurement. Clin Neuroradiol. https://doi.org/10.1007/s00062-019-00859-0

41. Kollmer J, Kästel T, Jende JME, Bendszus M, Heiland S (2018) Magnetization transfer ratio in peripheral nerve tissue: does it depend on age or location? Invest Radiol 53:397-402

42. Pridmore M, Castoro R, McCollum MS, Kang H, Li J, Dortch R (2020) Length-dependent MRI of hereditary neuropathy with liability to pressure palsies. Ann Clin Transl Neurol 7:15-25

43. Kollmer J, Sahm F, Hegenbart U et al (2017) Sural nerve injury in familial amyloid polyneuropathy. MR neurography vs clinicopathologic tools. Neurology 89:475-484

44. Kollmer J, Hund E, Hornung B et al (2015) In vivo detection of nerve injury in familial amyloid polyneuropathy by magnetic resonance neurography. Brain 138:549-562

45. Felisaz PF, Poli A, Vitale R et al (2019) MR microneurography and quantitative T2 and DP measurements of the distal tibial nerve in CIDP. J Neurol Sci 400:15-20

46. Pham M, Oikonomou D, Hornung B et al (2015) Magnetic resonance neurography detects diabetic neuropathy early and with Proximal Predominance. Ann Neurol 78:939-948

47. Ropele S, Filippi M, Valsasina P et al (2005) Assessment and correction of B1-induced errors in magnetization transfer ratio measurements. Magn Reson Med 53:134-140

48. Tofts PS, Steens SC, Cercignani M et al (2006) Sources of variation in multi-centre brain MTR histogram studies: body-coil transmission eliminates inter-centre differences. MAGMA 19:209-222

49. Berry I, Barker GJ, Barkhof F et al (1999) A multicenter measurement of magnetization transfer ratio in normal white matter. J Magn Reson Imaging 9:441-446

50. Barker GJ, Schreiber WG, Gass A et al (2005) A standardised method for measuring magnetisation transfer ratio on MR imagers from different manufacturers-the EuroMT sequence. MAGMA 18: 76-80

51. Kim SJ, Hong SH, Jun WS et al (2011) MR imaging mapping of skeletal muscle denervation in entrapment and compressive neuropathies. Radiographics 31:319-332

52. Chen Y, Haacke EM, Li J (2019) Peripheral nerve magnetic resonance imaging. F1000Res 8:1803

53. Sinclair CD, Morrow JM, Hanna MG et al (2012) Correcting radiofrequency inhomogeneity effects in skeletal muscle magnetisation transfer maps. NMR Biomed 25:262-270

Publisher's note Springer Nature remains neutral with regard to jurisdictional claims in published maps and institutional affiliations. 